\begin{tabular}{|c|c|}
\hline \multirow{3}{*}{ 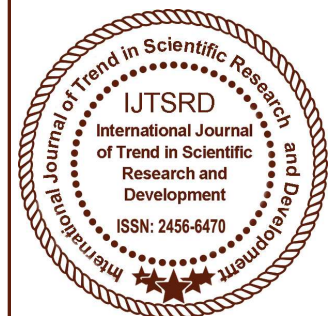 } & $\begin{array}{l}\text { International Journal of Trend in Scientific } \\
\text { Research and Development (IJTSRD) }\end{array}$ \\
\hline & International Open Access Journal \\
\hline & ISSN No: 2456 - 6470 | www.ijtsrd.com | Volume - 2 | Issue - 4 \\
\hline
\end{tabular}

\title{
Comparison Study Between Hydrogen Palladium Sensor and Hydrogen Manganese Dioxide Sensor
}

\author{
${ }^{1}$ Dr. Albashir Zomrawi , ${ }^{2}$ K. AlNaimee \\ ${ }^{1}$ Assistant Professor, College of Engineering, Karary University, Khartoum, Sudan \\ ${ }^{2}$ Istituto Nazionale diottica, LargoE. Fermi 6, 50125, Florence, Italy
}

\begin{abstract}
This research work oriented to compares between Palladium $(\mathrm{Pd})$ thin films sensor that have been prepared on glass substrates and hydrogen sensor using optical fiber, coated with Manganese dioxide $\left(\mathrm{MnO}_{2}\right)$ nanostructures. Palladium thin films prepared by vaporization deposition technique with annealing temperature around $600 \mathrm{C}^{0}$. Where, manganese dioxide, prepared on Optical Multimode Fiber (MMF)of $125 \mathrm{~m}$ in diameter as the transducing platform was tapered to $20 \mathrm{~m}$ to enhance the evanescent field of the light propagates in the fiber core.
\end{abstract}

The optical characteristics of the prepared films showed that they are highly sensitive, but their properties considerably vary when the measurements conducted in vacuum or in air. The response-recovery time of Pd materials to hydrogen gas is characterized to be extremely short.

The tapered optical fiber coated with $\mathrm{Pd} / \mathrm{MnO}_{2}$ nanograins was found to be sensitive towards hydrogen with different concentrations in synthetic air at $240^{\circ} \mathrm{C}$ operating temperature. The annealed sensor showed higher response and sensitivity compared with the as-prepared sensors when measured in the visible to near infrared optical wavelength range. The absorbance response of the annealed $\mathrm{Pd} / \mathrm{MnO}_{2}$ on fiber has increased to $65 \%$ compared to $20 \%$ for the as-prepared $\mathrm{Pd} / \mathrm{MnO}_{2}$ upon exposure to $1 \%$ Hydrogenin synthetic air.
Keywords: Manganese dioxide, Palladium, Semiconductor, Spectrometer, Sensor, Thin-Film

\section{INTRODUCTION}

Hydrogen gas occupies aboutmorethan $90 \%$ ofthe atmosphere. It is a highly flammable gas that burn at concentrations as low as $4 \%$ in air. It has a larger window of flammability in comparison to other natural gases such as gasoline, propane, ethane, methane, propylene, etc. The flammability limit of hydrogen is even times wider than methane. It is therefore, critical for a hydrogen sensor to have a wider measurement range for safety applications than most common fuels. Hydrogen is the lightest of elements and the smallest molecule; it, therefore, has the greatest tendency to leak ${ }^{[1]}$.

The use of hydrogen as a clean fuel in various applications requires practical and robust sensors to minimize risk of explosion associated with its volatile properties ${ }^{[2]}$. Thus, for a process safety application, a hydrogen leak can be more dangerous and its detection becomes more challenging than other flammable gases ${ }^{[1]}$.

\section{HYDROGEN SENSOR TECHNOLOGY}

Different of approaches that used to sense and detect hydrogen. Number of which are used in industry include the typical Gas Chromatography (GC), Mass Spectrometry (MS), Catalytic Bead (CB), and thermal conductivity. Semiconducting metal oxide and CB sensors are popular solid-state technologies, which employ heated catalysts to sense hydrogen. These 
sensors require heating to about $300^{\circ} \mathrm{C}$ to enable surface reactions that promote hydrogen sensing. Electrochemical sensors are based on known electrolytic reactions of hydrogen. Sensors based on catalytic combustion are generally nonspecific, electrochemical hydrogen sensors with liquid or solid type electrolytes having leakage issues. The hydrogen sensors based on thermal conductivity, CB, metal oxide, and electrochemical technologies require the presence of oxygen for sensor operation. Oxygen plays a crucial role in promoting the grain boundary formation in metal oxide sensors and electron transfer reactions in electrochemical sensors. The most promising solid-state technology is based on a hydrogen-specific material, palladium, which does not require oxygen for operation. Palladium-based sensors are gaining wide popularity in industry due to their reliability and high specificity to hydrogen. Any hydrogen sensor technology needs to satisfy the three basic requirements sensitivity, selectivity, and specificity ${ }^{[1]}$.

Number of traditional approaches can be used to detect Hydrogen, these may include:

i. Thermal Conductivity (TC): is the most widely applied measuring principle for the determination of hydrogen. "The measuring principle is based on the differences in thermal conductivity of the gases to be measured. A Thermal Conductivity Detector (TCD) measures the concentration of a gas in a binary gas mixture by measuring the thermal conductivity of the sample gas and comparing it to the thermal conductivity of a selected reference gas" [1].

ii. Gas Chromatography (GC): is also another widely applied measuring principle for hydrogen detection. "The disadvantages of GC are long response times (minutes) due to the chromatography, time-intensive sample preparation, consumable (carrier and calibration gases), and labor-intensive handling procedures. An advantage, however, is the ability to measure other gases such as nitrogen, oxygen, and carbon dioxide in the presence of hydrogen. But, this adds time to the total analysis" ${ }^{[1]}$.

A wide variety of solid-state sensors based on hydrogen-specific palladium, "Metal Oxide Semiconductor (MOS), CB, electrochemical, and Surface Acoustic Wave (SAW) technology are used in the industry for several years. Micro electromechanical systems (MEMS) and nanotechnology-based devices for the measurement of hydrogen are the recent developments. These developments are mainly driven by the demands of the fuel cell industry. Solid-state approaches are gaining rapid popularity within the industry due to their low cost, low maintenance, replacements, and flexibility of multiple installations with minimal labor $^{[1]}$.

Several types of palladium-based hydrogen sensors have been reported in the literature. The most notable ones are based on Pd thin-film resistors, FETs, Pd nanowires, $\mathrm{Pd}$ nanoparticle networks, Pd nanoclusters, and Pd nanotubes. Palladium Field-Effect Sensors: "Hydrogen sensors based on the "field effect" of palladium have been investigated extensively in the literature" [3] "The field effect results due to the rapid dissolution of hydrogen in the palladium surface arranged in a $\mathrm{Pd}-\mathrm{SiO} 2-\mathrm{Si}$ configuration. The sensor relies on an electric field resulting from the charge transfer between palladium and hydrogen on its surface. The FETs" [4] and "metal-insulatorsemiconductor (MIS)" [5] are the two major types of device structures that have been studied for palladium-based hydrogen sensing. Palladium is catalytically active, permeable to hydrogen, and can be readily used in FET and MIS devices.

i. Palladium-Based Resistors: "Thick- and thin-film palladium-based resistors have been reported for hydrogen sensing" [6]. The thick-film device uses printed palladium paste on a ceramic substrate in a four- resistor network. Two opposed resistors are covered to isolate them from the ambient atmosphere. "The exposure of the uncovered resistors to hydrogen results in a change in resistivity of the thick-film material and a shift in the balance point of the bridge, which can be scaled to the hydrogen concentration. The thin-film device is equivalent in design to the thick film; here, much thinner films (typically vacuum deposited) are used as the resistors. Thin-film palladium detectors have been prepared by depositing palladium through electron beam evaporation ${ }^{[7]}$, ' $R F$ magnetron sputtering, ${ }^{[8], ~ " m i c r o ~ c o n-t a c t ~ p r i n t i n g " ~[9], ~ a n d ~ w e t ~}$ electrochemistry. Most palladium resistors have fouling issues on the palladium surface due to impurities and pollutants in, or reaction with the air. The fouling on the palladium surface can be reduced by the addition of a second metal (alloy) to palladium. 
ii. Palladium-Coated Fiber Optic Sensors: A fiber optic hydrogen sensor consists of a palladium coating at the end of an optical fiber that senses the presence of hydrogen in air. "When the coating reacts with the hydrogen, its optical properties are changed. Light from a central electro-optic control unit is projected down the optical fiber where it is either reflected from the sensor coating back to central optical detector or is transmitted to another fiber leading to the central optical detector. A change in the reflected or transmitted intensity indicates the presence of hydrogen. The fiber optic detector offers inherent safety by removing all electrical power from the test sites and reduces signal-processing problems by minimizing electromagnetic interference. The fiber optic hydrogen sensors can be fabricated using a palladium-coated single-mode tapered optical fiber" ${ }^{10]}$. The attenuation change of the fiber-mode when the device was exposed to hydrogen is used to detect and measure hydrogen concentration in gaseous atmospheres.

\section{HYDROGEN SENSORCOMPONENS}

PALLADIUM

Hydrogen palladium thin films sensor consists basically on seven components include; chamber, white photo diode, spectrometer, vacuum unit, gauge pressure, cylinder of hydrogen gas, and computer. Figure (1) and figure (2) below illustrate sensor component and sensor setup successively. A sample of palladium coated glass slide sensor should be prepared first to detect hydrogen, and should be tested by X-ray to cheek its crystallization ${ }^{[11]}$.

The main sensor components are;

a. The coated glass substrate (Palladium thin films): should be placed inside the developed polymer square test chamber of $50 \mathrm{~mm}$ square base and of $75 \mathrm{~mm}$ height with the top removable cover. The effective volume of the chamber is $187500 \mathrm{~mm}^{3}$; it has an inlet to allow the test gas to flow in ${ }^{[11]}$.

b. White photo diode: is the light source used in the sensor.

c. CCS Spectrometer: used to measure transmitted.

d. Vacuum unit: to evacuate the test chamber.

e. Gauge pressure: to measure the current pressure of the chamber. f. Cylinder of hydrogen gas of a known concentration:

to flow through the test chamber during measurement.

g. Computer: to process the recorded signal.

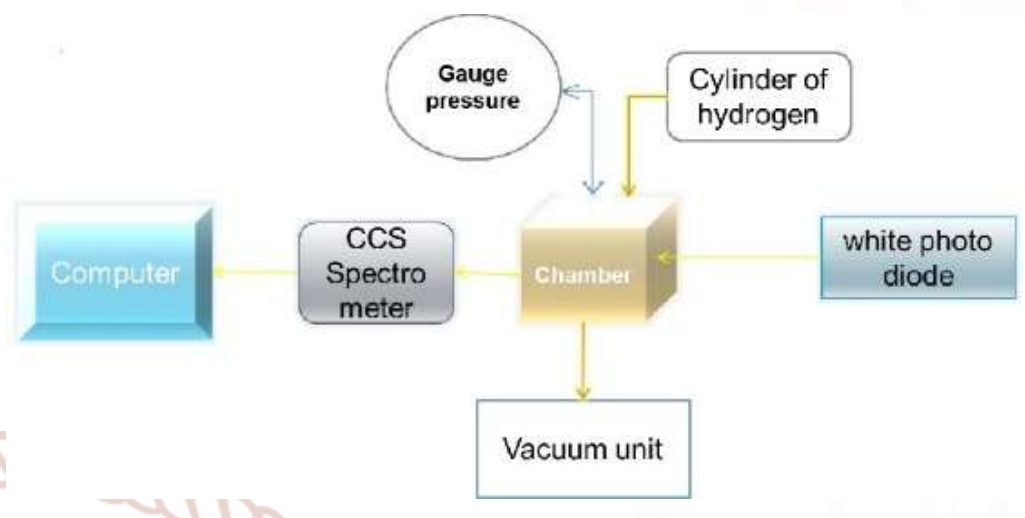

Fig: 1 Sensor components

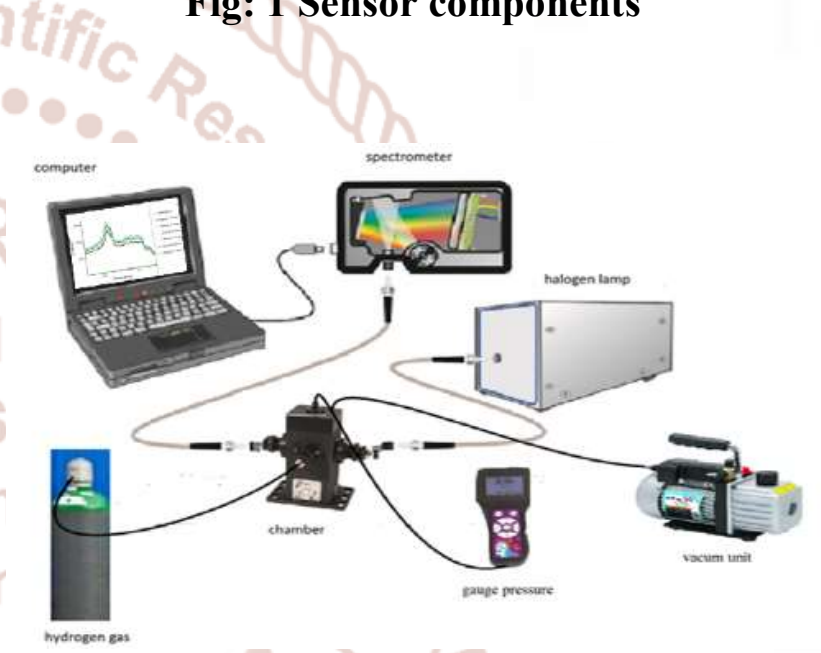

Fig: 2 Sensor setup

\section{SETUPOF HYDROGEN MANGANESE DIOXIDE SENSOR}

The optical hydrogen gas sensing setup comprised of tungsten halogen light source modelled HL-2000, Ocean Optics USA which exhibit wavelength range of $360 \mathrm{~nm}$ to $2500 \mathrm{~nm}$. Optical absorbance spectrum was monitored via spectrophotometer (USB4000 VISNIR, Ocean Optics USA) with spectral response ranged between $200 \mathrm{~nm}$ to $1100 \mathrm{~nm}$ of wavelength. The light source was coupled into the MMF by using a standard SMA optical cable $(600 \mathrm{~nm}$ in core diameter) for light transmission. The MMF termination is FC/PC type. Hence, an SMA-FC adapter was used to couple the SMA cable to the MMF. From the adapter data sheet, typical insertion loss for mating FC/PC terminated MMF to SMA terminated cable is $1.4 \mathrm{~dB}$. The calculated coupling efficiency based on this insertion loss was $99.6 \%$. The $\mathrm{MnO}_{2}$-coated on tapered optical fiber sensor was 
placed in the sealed gas chamber as demonstrated in Figure (3). The gas purging into the chamber was controlled by a computer-controlled mass flow controller which regulates gas flux at a flow rate of $200 \mathrm{sccm}$. In order to obtain the desired concentration of hydrogen gas, pure synthetic air was used to dilute the hydrogen gas to concentration varied from $0.125 \%$ to $1.0 \%$. The absorbance measurement and dynamic response of cumulative absorbance were monitored while purging alternately hydrogen gas and pure synthetic air, respectively. The chamber is also equipped with heater plate which can be varied by dc voltage supply as a source of operating temperature [12].

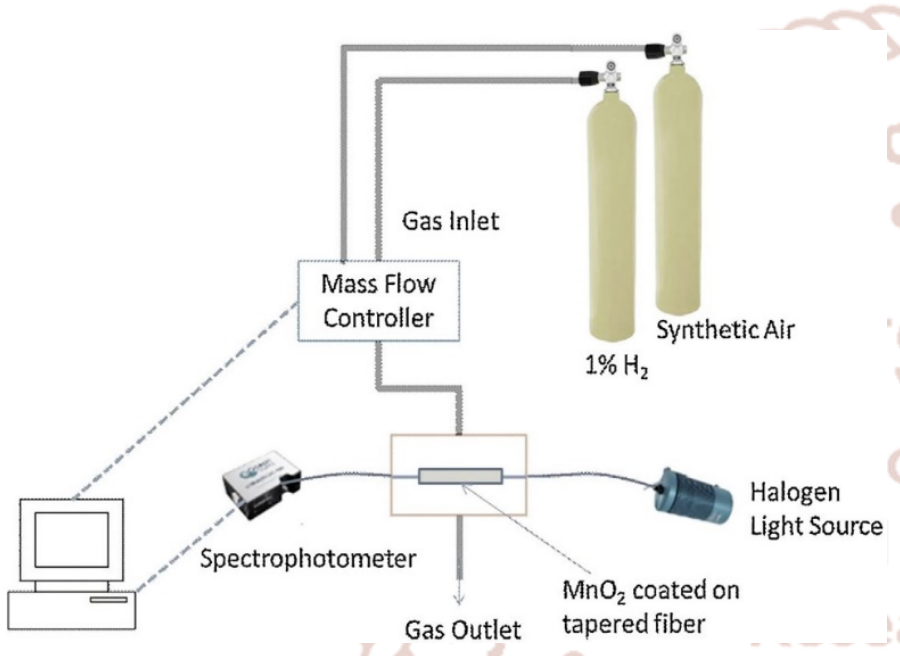

Fig: 3 Gas sensing experimental setup

\section{MEASUREMENTS AND RESULTS}

Tow testes were carried out to examine the palladium thin film sensor. In both tests, results were obtained through adopting the following steps:

Opening the test chamber to place the palladium thin film sensor on the sensor holder and close it. The necessary light source is then directed by optical fiber and allowed to pass through the sample to the spectrometer. The rotary pump is then switched on to evacuate the test chamber to about -0.7 bars. Next, the hydrogen gas of a known concentration allowed passing from the cylinder through the special in letto the test chamber by opening the cylinder valve. Test chamber pressure should be measured by observing the gauge pressure. Spectrometer is then detects and analyzes transmitted signals and sends the data to the computer.

Numbers of measurements were carried out in different pressures. Six readings were observed for each sample test including pressures of $-0.6,-0.5,-0.4$,
$-0.3,-0.2$ and -0.1 bar. Frequency against transmitted light intensity graphs were produced for each reading. The six graphs were then combined together in one diagram with a background graph representing -0.7 pressure without Hydrogen. Figure (4) and figure(5)below represent the resultant combined graphs for both sample tests ${ }^{[11]}$.

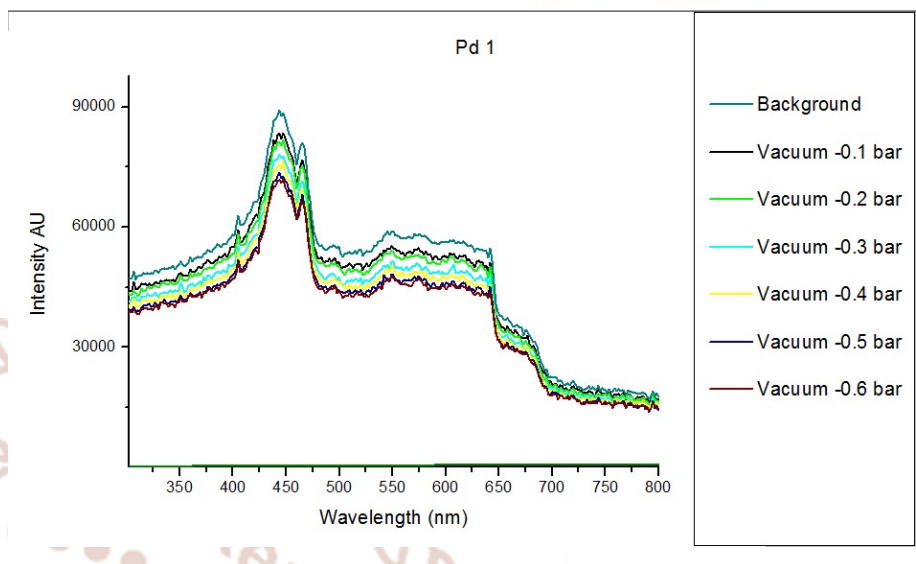

Fig: 4.Combined graphs of the palladium thin film sample one (Pd 1) with a numbers of measurements were carried out indifferent pressures. Six readings were observed for each sample test including pressures of $-0.6,-0.5,-0.4,-0.3,-0.2$ and -0.1 bar.

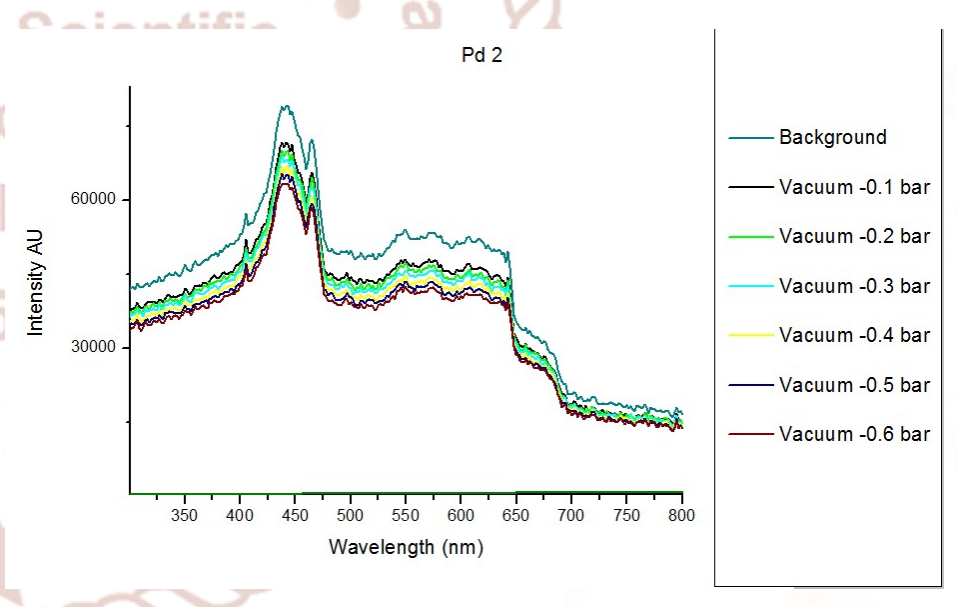

Fig: 5.Combined graphs of the palladium thinfilm sample two (Pd 2)

From the figures obtained above it can be noted that by increase of concentration of hydrogen gas in the test chamber, the transmitted light is also increase which, means that the palladium thin film sample successfully detect hydrogen gas. And it can obviously note that the suitable wavelength that can be used to detect hydrogen gas is a visible band that located in the regionof500-650nm.

In the Manganese dioxide sensor the gas absorbance response was carried out at operating temperature of $240^{\circ} \mathrm{C}$. No response was recorded below this 
operating temperature, possibly because of a slow chemical activation between adsorbed gas molecules and the sensing layer ${ }^{[13]}$. Figure (6) demonstrates the absorbance response of $\mathrm{Pd} / \mathrm{MnO}_{2}$ coated optical fiber sensor for as-prepared and annealed samples towards $1 \% \mathrm{H}_{2}$. The integration of $\mathrm{Pd}$ on $\mathrm{MnO}_{2}$ layer is important to catalyze the gas dissociation and alter the layer optical properties when interact with $\mathrm{H}_{2}{ }^{[14]}$. $\mathrm{Pd}$ has the ability to highly absorb $\mathrm{H}_{2}$ at almost900 times of its own volume ${ }^{[15]}$. When both as-prepared and annealed samples were exposed to synthetic air and $1 \%$ of $\mathrm{H}_{2}$ gas at elevated temperature of $240^{\circ} \mathrm{C}$, a distinctive change of absorbance magnitude over the range of $500-800 \mathrm{~nm}$ was observed. However, the annealed sensor gives higher magnitude of absorbance as compared to the as-prepared when exposed to $\mathrm{H}_{2}$. The absorbance change in the $\mathrm{MnO}_{2}$ layer can be explained with the following mechanism. During $\mathrm{H}_{2}$ adsorption onto the sensing layer, the dissociation of $\mathrm{H}_{2}$ molecules onto $\mathrm{Pd}$ resulting in the generation of $\mathrm{H}+$ ions and electrons. Ions of $\mathrm{H}+$ were spilled over onto the $\mathrm{MnO}_{2}$ nanograins layer which then reacted with chemisorbed oxygen $\left(\mathrm{O}_{2}-\right.$ and $\left.\mathrm{O}_{-}^{-}\right)$resulting in production of $\mathrm{H}_{2} \mathrm{O}$ molecules ${ }^{[13]}$. The generated electrons reduced $\mathrm{Mn} 4+$ in the center of the $\mathrm{MnO} 2$ crystal lattice to $\mathrm{Mn} 3+{ }^{[16]}$. Hence, the film refractive index and its absorbance changed. Upon exposure to synthetic air, the adsorbed oxygen as well as the desorbed $\mathrm{H}+$ ions in/out of the sensing layer returned the layer absorbance spectrum to its original baseline.

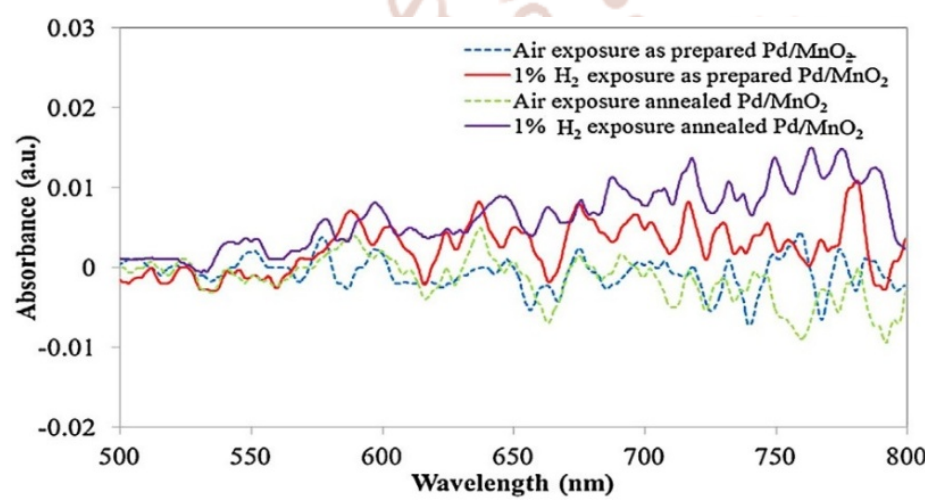

Fig. 6: Absorbance versus optical wavelength of $\mathrm{Pd} / \mathrm{MnO} 2$ sensor for as-prepared and annealed samples exposed to $1 \%$ concentration of $\mathrm{H} 2$ in synthetic air at $240 \circ \mathrm{C}$.

\section{CONCLUSION}

This work is a comparison study between two developed hydrogen sensors. The first depended on the Palladium coated glass thin film and the other depended on optical fiber coated with manganese dioxide. From the measurements carried out and results obtained, it can be concluded with that both sensor efficiently detected hydrogen and electromagnetic interference free.

The Hydrogen palladium thin films sensor has rapid response to low hydrogen concentration, also it has a wide dynamic range specifically in 500-650nm range. Moreover, the sensor can be used in the room temperature.

On the other hand, $\mathrm{Pd} / \mathrm{MnO}_{2}$ coated optical fiber sensor is sensitive at elevated temperature of $240^{\circ} \mathrm{C}$ therefore, it can be used in the hot areas but, it is more expensive compared with Palladium coated glass thin film sensor.

\section{REFERENCES}

1. Ram B. Gupta. "Hydrogen Fuel Production, Transport, and Storage". Edited by Taylor \& Francis Group, LLC-2009 .

2. V.E. Bochenkov, G.B. Sergeev, Sensitivity, selectivity, and stability of gas-sensitive metaloxide, Nanostructures 3 (2010) 31-52.

3. Robins, I., Ross, J.F. and Shaw, J.E.A., "The logarithmic response of palladium-gate metal" insulator-silicon field-effect to hydrogen, Journal of Applied Physics, 60(2), 843, 1986.

4. Cassidy, J., Pons, S. and Janata, J., "Hydrogen response of palladium coated suspended gate field effect transistor", Analytical Chemistry, $58(8), 1757,1986$.

5. Salomonsson, A., Eriksson, M. and Dannetun, H., "Hydrogen interaction with platinum and palladium metal-insulator-semiconductor devices", Journal of Applied Physics, 98, 014505 , 2005.

6. Pitts, J.R., Liu, P., Lee, S.-H. and Tracy, C.E., "Interfacial instability in hydrogen sensors", Pro- ceedings of the 2000 DOE Hydrogen Program Review, Available at http://www1.eere. energy.gov/ hydrogenandfuelcells/pdfs/28890g.pdf.

7. Thomas, R.C. and Hughes, R.C., "Sensors for Detecting Molecular Hydrogen Based on Pd Metal Alloys", Journal of Electrochemical Society, 144(9), 3245, 1997. 
8. Oh, Y.-s., Hamagami, J.-i., Watanabe, Y., Takata, M. and Yanagida, H., "Palladium thin fi Im hydrogen detector", Journal of the Ceramic Society of Japan, 101(1174), 618-620, 1993.

9. Wolfe, D.B., Love, J.C., Paul, K.E., Chabinyc, M.L. and Whitesides, G.M., "Fabrication of palladium- based microelectronic devices by microcontact printing", Applied Physics Letters, 80, 2222, 2002.

10. Villatoro, J., D 'iez, A., Cruz, J.L. and Andr 'es, M.V., "Highly sensitive optical hydrogen sensor using circular Pd-coated single mode tapered fiber, Electron”, 37, 1011, 2001.

11. Al bashir Zomrawi, A. M. Awadelgied, S. F. Abdalah, K. Al Naimee, "Palladium Film Chemical Sensor", International Journal of Innovation in Science and MathematicsVolume 2, Issue 2, ISSN (Online): 2347-9051.

12. Nor Akmar Mohd Yahya, Mohd Rashid Yusof Hamid, Siti Azlida Ibrahim, , Boon Hoong Ong, Norizah Abdul Rahman, Ahmad Rifqi Md Zain, Mohd Adzir Mahdi, Mohd Hanif Yaacob, "H2 sensor based on tapered optical fiber coated with $\mathrm{MnO} 2$ nanostructures" Sensors and Actuators B 246 (2017) 421-427.

13. A. Sanger, A. Kumar, A. Kumar, R. Chandra, Highly sensitive and selective hydrogen gas sensor using sputtered grown Pd decorated $\mathrm{MnO} 2$ nanowalls, Sens. Actuators B: Chem. 234 (2016) $8-14$, http://dx.doi.org/10.1016/j.snb. 2016.04.152.

14. M.H. Yaacob, A.Z. Sadek, K. Latham, K. Kalantar-zadeh, W. Wlodarski, Procedia chemistry optical H2 sensing performance of anodized nanoporous $\mathrm{TiO} 2$ thin films, PROCHE 1 (2009) $951-$ 954,http://dx.doi.org/10.1016/j.proche.2009.07.

15. Manchester, A. San-Martin, J. Pitre, The H-Pd (Hydrogen-Palladium) system, J. Phase Equilibria 15 (1994) 62-83.

16. Y. Zhang, L. Zhang, J. Deng, S. Xie, H. Yang, Y. Jiang, H. Dai, Synthesis, characterization, and catalytic properties of $\mathrm{MnO}$ x/SBA-16 for toluene oxidation, Proc. Mat. Sci. Energy Eng. (2014). 